\section{Toward a Personalized Approach in the Treatment of Salivary Ductal Carcinoma}

\author{
Frederick D. Tsai, MD, PhD, and Robert I. Haddad, MD
}

Establishing guidelines for the management of rare and aggressive tumors poses a distinct challenge. Among these tumors, salivary gland cancers, which occur in roughly 1 per 100,000 people annually worldwide, lack robust evidence from clinical trials to support a standard of care for treating recurrent and/or metastatic disease. In the absence of largescale studies or expert consensus, reports from case studies that show promising responses and a better understanding of the molecular biology of salivary tumors provide some framework for management.

Recent attempts to better characterize the biology of salivary gland carcinomas have been fruitful through immunohistochemical detection of targetable receptor expression. The observation that salivary ductal carcinoma (SDC) histologically resembles breast adenocarcinoma led to the finding of HER2 expression in a subset of SDC, which responded well to inhibition with trastuzumab. ${ }^{1,2}$ Indeed, the standard approach to treating patients with SDC at Dana-Farber Cancer Institute incorporates routine testing of HER2 status and combines chemotherapy with trastuzumab in a treatment program that is delivered over a 12 -month period. ${ }^{2}$ Our approach is applicable to patients treated in a curative, postoperative setting (with radiation therapy) or in the incurable, recurrent, and metastatic settings. The contribution of chemotherapy to this regimen is unknown, and the rarity of this disease makes it unlikely that a study addressing this question will ever be performed.

Immunohistochemical detection of androgen receptor expression has also led to similar, encouraging results with androgen deprivation therapy (ADT) for recurrent or disseminated disease, with a case series of 10 patients with androgen receptor-positive SDC demonstrating benefit from hormonal therapy. ${ }^{3,4}$ This was followed by a nationwide case series of 35 patients in the Netherlands, $18 \%$ of whom had a partial response and $32 \%$ experienced stable disease, leading to a 12 -month increase in median overall survival in this cohort. ${ }^{5}$ These results have provided a new avenue of treatment for SDC.

Elsewhere in this issue, Lin et al demonstrate the utility of next-generation sequencing (NGS) in deriving molecular targets for SDC, with good response. They describe a patient with widely metastatic SDC, who, on targeted NGS, was found to have a BRAF V600E mutation. The patient was treated with dabrafenib and trametinib as firstline systemic therapy, and experienced 13 months of stable disease before progression was noted on restaging scans. NGS and analysis of circulating tumor DNA were subsequently used to guide clinical decision-making, although no actionable mutations were found. Reanalysis of the tumor revealed positive androgen receptor expression, and the patient was started on ADT; however, after 1 month and disease progression, he ultimately chose hospice care.

Use of NGS has changed the landscape of tumor profiling and led to a shift in paradigm in disease classification that previously was based more on morphology and histochemical markers. More importantly, identification of common mutations and pathogenic alterations in shared signaling pathways allows for the use of rational targeted therapy. In the absence of available clinical trials and randomized controlled studies to drive clinical decision-making for rare tumors such as SDCs, the analogous use of known molecular inhibition for these pathways may provide an alternative paradigm for treatment. HER2 inhibition and androgen deprivation have been well studied and characterized in breast and prostate cancers, and their successful use in SDC indicates that, although they arise in different tissue types, shared biologic features allow SDC to respond. BRAF and MEK inhibition, famously studied and described to be highly effective in melanoma, may also

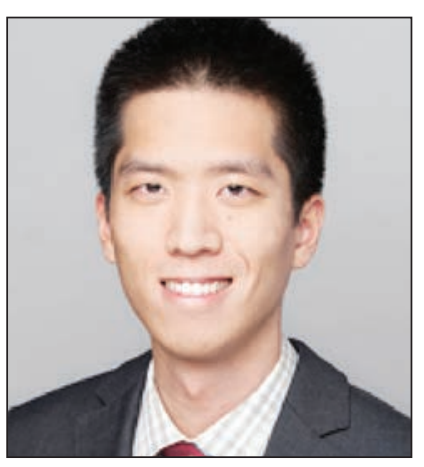

Frederick D. Tsai, MD, PhD

Frederick D. Tsai, MD, PhD, is a Clinical Fellow in the Department of Medical Oncology at DanaFarber Cancer Institute and at Harvard Medical School. 


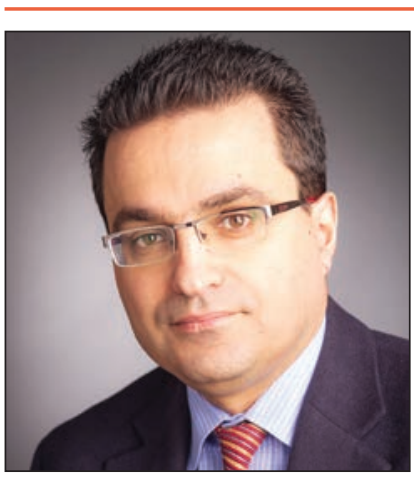

Robert I. Haddad, MD

Robert I. Haddad, MD, is Chief, Division of Head and Neck Oncology and Professor of Medicine in the Department of Medical Oncology at Dana-Farber Cancer Institute and Harvard Medical School. play a similar role in SDC. What more might we find? Further genomic alterations and copy number changes in other signaling pathways, including PI3K/AKT/mTOR, RAS, and cyclin D1/CDK, have increasingly been described for SDC, and although they are of uncertain clinical significance, they provide potential targets for molecular therapy for a disease historically unresponsive to conventional cytotoxic chemotherapy. ${ }^{6}$

The plural of anecdote is not data, and caution should be exercised in generalizing these findings toward a standard of care. However, in the absence of robust clinical trial data and with the tremendous challenges in conducting large-scale trials in rare tumors, these case reports provide a rubric for the management of SDCs-and by extension other rare tumors - with molecular profiling and NGS in the upfront setting to help guide clinical decision-making and possibly improve patient outcomes.

\section{References}

1. Glisson B, Colevas AD, Haddad R, et al. HER2 expression in salivary gland carcinomas: dependence on histological subtype. Clin Cancer Res 2004;10:944-946.

2. Limaye SA, Posner MR, Krane JF, et al. Trastuzumab for the treatment of salivary duct carcinoma. Oncologist 2013;18:294-300

3. Jaspers HC, Verbist BM, Schoffelen R, et al. Androgen receptor-positive salivary duct carcinoma: a disease entity with promising new treatment options. J Clin Oncol 2011;29:e473-476.

4. Dalin MG, Watson PA, Ho AL, Morris LG. Androgen receptor signaling in salivary gland cancer. Cancers (Basel) 2017;9.

5. Boon E, van Boxtel W, Buter J, et al. Androgen deprivation therapy for androgen receptor-positive advanced salivary duct carcinoma: a nationwide case series of 35 patients in The Netherlands. Head Neck 2018;40:605-613.

6. Schmitt NC, Kang H, Sharma A. Salivary duct carcinoma: an aggressive salivary gland malignancy with opportunities for targeted therapy. Oral Oncol 2017;74:40-48. 